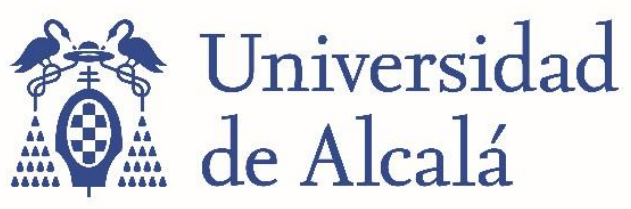

BIBLIOTECA

Document downloaded from the institutional repository of the University of Alcala: http://dspace.uah.es/

This is a postprint version of the following published document:

Caucheteur, C., Bigourd, D., Hugonnot, E., Szriftgiser, P., Kudlinski, A., et al., "Chirped pulse amplification in a fiber optical parametric amplifier", Proc. SPIE7728, Nonlinear Optics and Applications IV, 77280J (June 04, 2010); doi:10.1117/12.854368;

Available at http://dx.doi.org/10.1117/12.854368

Copyright 2010 Society of Photo Optical Instrumentation Engineers. One print or electronic copy may be made for personal use only. Systematic electronic or print reproduction and distribution, duplication of any material in this paper for a fee or for commercial purposes, or modification of the content of the paper are prohibited.

(Article begins on next page)

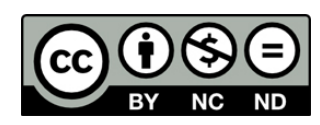

This work is licensed under a

Creative Commons Attribution-NonCommercial-NoDerivatives

4.0 International License. 


\title{
Chirped pulse amplification in a fiber optical parametric amplifier
}

\author{
C. Caucheteur*a, D. Bigourd ${ }^{\mathrm{b}}$, E. Hugonnot ${ }^{\mathrm{b}}$, P. Szriftgiser ${ }^{\mathrm{c}}$, A. Kudlinski ${ }^{\mathrm{c}}$, M. González-Herráez ${ }^{\mathrm{d}}$, \\ A. Mussot ${ }^{\mathrm{c}}$ \\ ${ }^{a}$ Electromagnetism and Telecommunication Unit, Faculté Polytechnique, Université de Mons, \\ Boulevard Dolez 31, 7000 Mons, Belgium; \\ ${ }^{b}$ Centre d'Etudes Scientifiques et Techniques d'Aquitaine, Commissariat à l'Energie Atomique, \\ B.P. 2, 33114 Le Barp, France; \\ 'IRCICA, FR CNRS 3024, Laboratoire PhLAM, UMR CNRS 8523, Université des Sciences et \\ Technologies de Lille, 59655 Villeneuve d'Ascq Cedex, France; \\ ${ }^{\mathrm{d}}$ Department of Electronics, Universidad de Alcalá, 28805 Alcalá de Henares, Spain
}

\begin{abstract}
Fiber optical parametric amplifiers (FOPAs) have attracted considerable attention during the last decade because of their broad bandwidth, high gain and wavelength-flexibility. In comparison to cumbersome bulky systems, they bring the advantages of all-fiber systems, i.e. reliability, long-term stability and compactness. FOPAs rely on the third-order susceptibility and are characterized by a quasi-instantaneous nonlinear response that involves pump, signal and idler waves. Chirped pulse amplification (CPA) allows to get a high energy amplification and its realization in FOPAs would increase the overall performances of these amplifiers. Such an experimental demonstration has never been reported in the past. In this work, we show for the first time the experimental feasibility of fiber-based optical parametric chirped pulse amplification (FOPCPA) with an all-fibered setup. The stretching/compression stages are realized with a single linearly chirped fiber Bragg grating (LCFBG) used in both directions while the amplification is performed in a CW-pumped FOPA that uses 500 meters of highly nonlinear fiber (HNLF). Fourier transform limited optical pulses at $1550 \mathrm{~nm}$ are stretched from 6 ps to $70 \mathrm{ps}$ and then amplified by $22 \mathrm{~dB}$ without any spectral or temporal distortions. Experiments are confirmed by simulations carried out by numerical integration of the nonlinear Schrödinger equation with parameters matching those of the experimental setup. For simplicity, this first experimental demonstration is realized in the telecommunication window. By using photonic crystal fibers, one can move the working wavelength around $1 \mu \mathrm{m}$.
\end{abstract}

Keywords: Nonlinear optics, fibers, Bragg gratings, parametric oscillators and amplifiers.

\section{INTRODUCTION}

Optical parametric amplification (OPA) is a well-known process which conventionally consists in amplifying a weak signal beam in a suitable second-order nonlinear medium by a high intensity pump beam. Combined with chirped pulse amplification (CPA) ${ }^{1}$ and termed as optical parametric chirped pulse amplification (OPCPA), it has soon been recognized to be a very attractive technique for high energy amplification of ultrashort pulses ${ }^{2,3}$. In few millimeter-long crystals, it can be used to amplify broadband pulses with very high gain, high conversion efficiency, low B-integral, good beam quality and high temporal contrast ${ }^{4}$. It has been used to amplify few-cycle pulses up to $90 \mathrm{~mJ},{ }^{5}$ to produce high energy femtosecond pulses ${ }^{6}$ and to seed high-power Nd:glass laser chains ${ }^{7,8}$ for ultra-high intensity experiments ${ }^{9}$. However, these bulk systems are quite cumbersome and complex to implement in experiments. In order to benefit from the ease of use of all fiber systems as well as their reliability, long-term stability and compactness, we propose to investigate the possibility to realize an all-fiber OPCPA system. Such a system was first proposed and numerically investigated by Hanna et $a l^{10}$. However, no experimental demonstration of this concept has been done yet. During the last decade, fiber-optic parametric amplifiers (FOPAs) have focused the attention of many researchers in the field of optical telecommunications because of their broad bandwidth, high gain, wavelength flexibility and wavelength conversion properties ${ }^{11,12}$. These fiber amplifiers rely on the third-order nonlinearity but have important similarities with OPAs. Their nonlinear response is quasi-instantaneous and a conjugate wave, the idler, is generated during the amplification process ${ }^{11,12}$.

*christophe.caucheteur@umons.ac.be; phone +32 65 374149; fax +32 65 374199; www.telecom.fpms.ac.be 
In this work, we experimentally demonstrate for the first time to our knowledge a fiber-based optical parametric chirped pulse amplification (FOPCPA) in an all-fiber configuration. Both the stretching and compression stages are realized with the same linearly chirped fiber Bragg grating (CFBG) and the pulse amplification is performed in a continuous-wave (CW)-pumped FOPA. For the sake of simplicity, this first experimental demonstration is realized in the telecommunication window around $1.5 \mu \mathrm{m}$. However, there is in principle no limitation to develop a similar system around $1 \mu \mathrm{m}$ by using photonic crystal fibers as gain medium ${ }^{13}$ and suitable fiber components.

The remaining of the paper is organized as follows. Section 2 describes the experimental setup while section 3 focuses on the experimental results. The latter are confirmed by numerical simulations obtained by integration of the generalized nonlinear Schrödinger equation (GNLSE).

\section{EXPERIMENTAL SETUP}

The experimental setup is represented in Fig. 1. A tunable CW laser source is phase-modulated to avoid stimulated Brillouin scattering (SBS), and amplified with an erbium-doped fiber amplifier (EDFA). A tunable spectral filter (100 $\mathrm{GHz}$ at full width at half maximum (FWHM)) is placed at the output to remove the excess of amplified spontaneous emission (ASE). The only difference with most of the FOPA setups is that we used a noise microwave source to drive the phase modulator rather than random bit sequences. By this way, SBS is still efficiently suppressed and the setup is drastically simplified without modifying the FOPA performances ${ }^{14}$. Here, the pump spectral bandwidth is extended up to $2.6 \mathrm{GHz}$, leading to a measured SBS threshold of $29 \mathrm{dBm}$. To prevent any damage on the filters, the pump power is maintained below this limit, at $27.6 \mathrm{dBm}(575 \mathrm{~mW})$. The pump wavelength is centered at $1559.59 \mathrm{~nm}$, just above the zero-dispersion wavelength $\left(\lambda_{0}=1554.6 \mathrm{~nm}\right)$ of the highly nonlinear fiber (HNLF) used as amplifying medium (provided by Sumitomo). The fiber length is $500 \mathrm{~m}$, the dispersion slope is $0.026 \mathrm{ps} / \mathrm{nm} / \mathrm{km}$, the nonlinear coefficient is $13.3 \mathrm{~W}^{-1} \cdot \mathrm{km}^{-1}$ and the attenuation is $0.6 \mathrm{~dB} / \mathrm{km}$ at $1550 \mathrm{~nm}$.

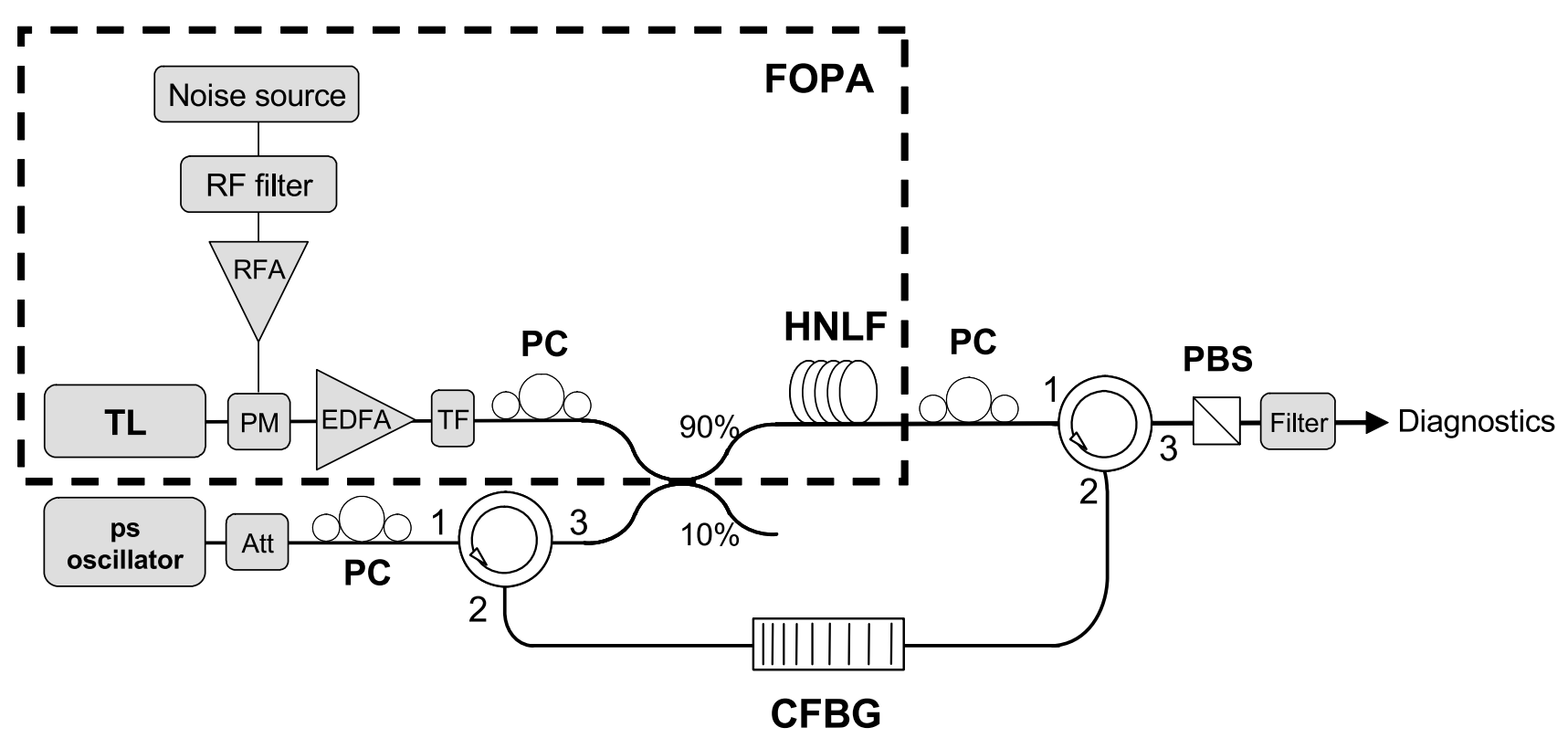

Fig. 1. Experimental set-up of FOPCPA. TL : tunable laser, PM : phase modulator, RFA : Radiofrequency amplifier, EDFA : Erbium doped fiber amplifier, TF : tunable filter, PC : polarization controller, HNLF: highly non-linear fiber, CFBG: chirped fiber Bragg grating, PBS: polarizing beam splitter.

Another originality of the setup resides in the use of a single CFBG to stretch and compress the signal pulses, as proposed in ref. [15] for optical delay line purposes. A $15 \mathrm{~cm}$-long grating was written into a hydrogen-loaded standard 
single mode fiber by means of the phase mask technique using a continuous-wave frequency-doubled argon-ion laser emitting at $244 \mathrm{~nm}$. The obtained grating is characterized by a chromatic dispersion of about $350 \mathrm{ps} / \mathrm{nm}$. Its refractive index profile was apodized to minimize the ripple on the group delay curve. This one reaches less than a few percents of the mean group delay value. After the inscription process, the grating was annealed during 2 days at $100{ }^{\circ} \mathrm{C}$ to stabilize its spectral properties.

Fig. 2 depicts the CFBG reflected spectrum and the corresponding group delay evolution when the light is injected through the short-wavelengths port. These ones are both measured using an optical vector analyzer from LUNA technologies with a wavelength resolution set to $3 \mathrm{pm}$.

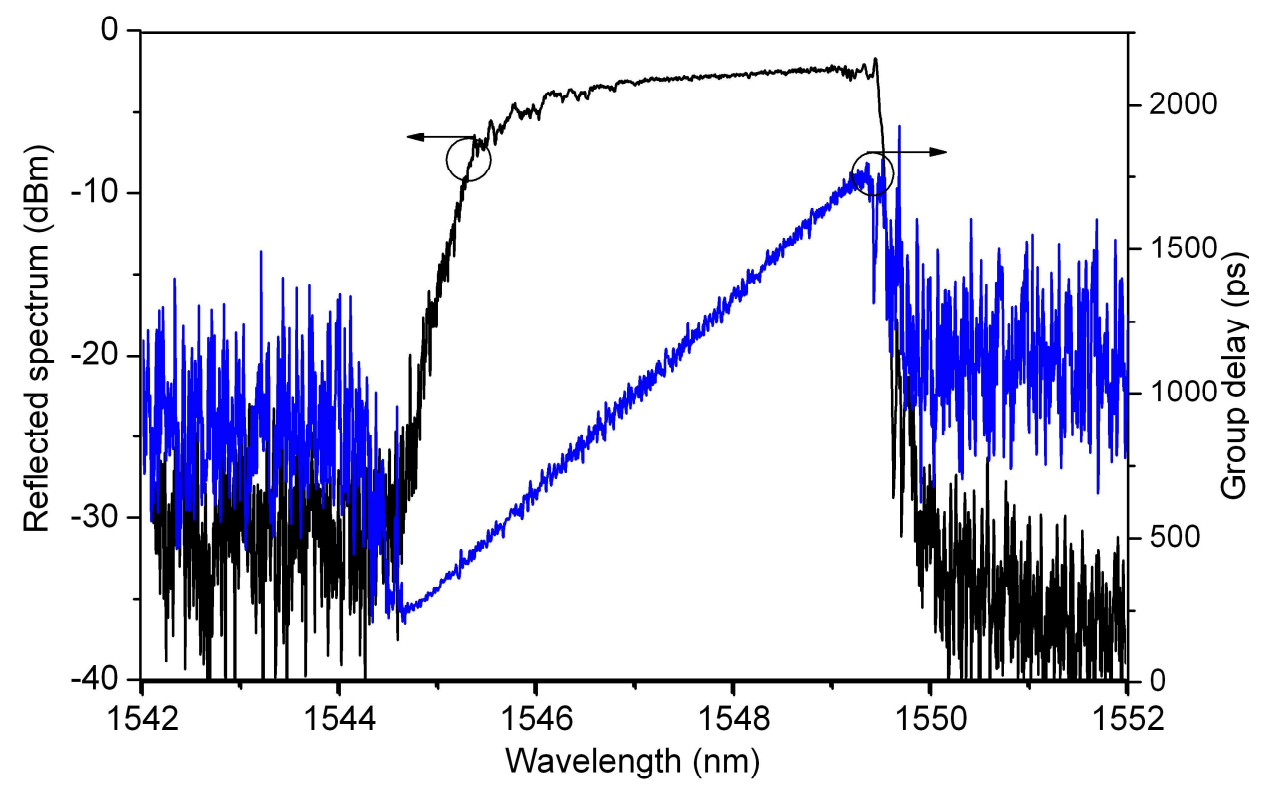

Fig. 2. Reflected amplitude spectrum and associated group delay evolutions (short wavelengths port) of the used CFBG.

In our setup, the signal pulses are obtained from a picosecond oscillator which delivers 6.4 ps full-width at halfmaximum (FWHM) transform-limited pulses. Prior to be amplified, the pulses are sent to the short wavelength port of the CFBG. As a result, they are affected by the CFBG chromatic dispersion. The reflected pulses are then stretched to an estimated FWHM of 190 ps so that they are about 30 times larger than the initial duration. The chirped signal and the pump are combined via a $90 / 10$ fiber coupler ( $90 \%$ of the pump goes into the HNLF) and are launched inside the HNLF where the amplification process occurs. After propagation in the HNLF, the amplified pulses are filtered and again fed into the CFBG, this time through the long-wavelengths port. Therefore, assuming that no extra chirp is added during the propagation through the HNLF, this setup ensures a perfect matching between stretching and compression (the chirp seen from both sides of the CFBG should be exactly the opposite) and the output pulses present the same shape as the input ones.

Two polarization controllers are used to achieve orthogonal states of polarization between the beams getting out of the short-wavelengths port and entering in the long-wavelengths one. Indeed, since the CFBG is not perfectly $100 \%$ reflective, a small part of the pulses launched in the short-wavelengths port are transmitted and could interfere with the useful signal getting out of the setup. This parasitic signal is removed by using a polarizing beam splitter placed behind the second circulator and aligned along the polarization state of the recompressed signal. Since the central wavelength of the CFBG cannot be easily tuned (mechanical or thermal actuations were preferably not implemented to avoid any undesired wavelength shift), we have adjusted the wavelength and the power of the FOPA pump in order to achieve a perfect phase matching around the CFBG central wavelength $(1546.6 \mathrm{~nm})$. This choice combines the advantages of achieving high and nearly flat gain over the spectral width of the signal $(0.55 \mathrm{~nm})$. This can be seen in Fig. 3 which depicts the parametric fluorescence spectrum (solid line) and the input signal spectrum (dotted line) recorded using an optical spectrum analyzer ANDO AQ6317B. 


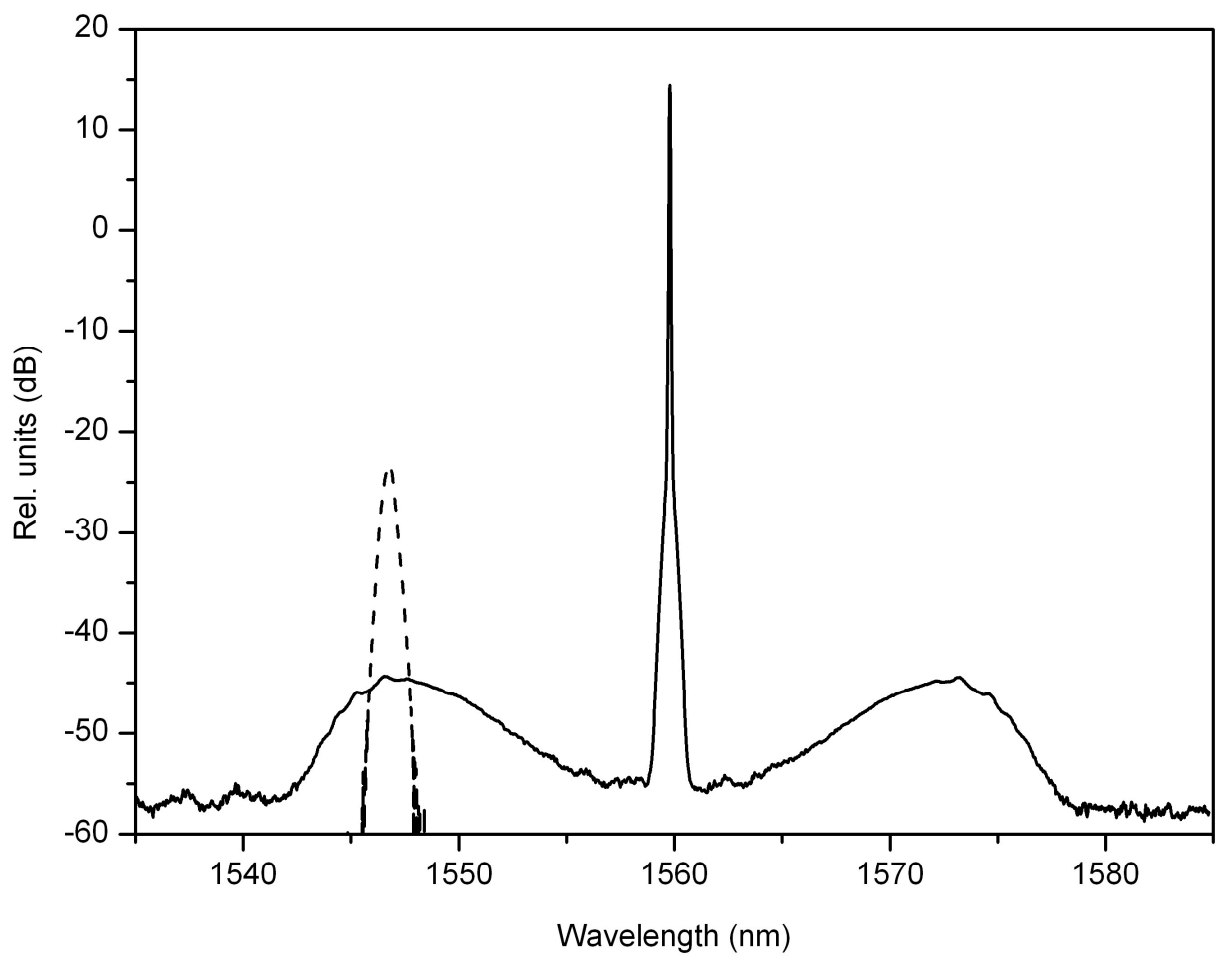

Fig. 3. Optical spectrum measured at the FOPA output with signal switched off (solid line). The initial signal spectrum is superposed as a guideline (dashed line).

\section{RESULTS AND DISCUSSION}

First of all, we have recorded the evolution of the gain as a function of the input signal energy. Corresponding measurements are represented in Fig. 4 in circles. The signal gain starts at $24.8 \mathrm{~dB}$ for the lowest energy pulses and rapidly drops beyond $100 \mathrm{fJ}$ pulses, to reach a gain of $12 \mathrm{~dB}$ for an input signal energy of $1.5 \mathrm{pJ}$. The gain obtained for smallest signals $(\approx 25 \mathrm{~dB})$ in the linear regime of the amplifier is very close to the gain of a weak monochromatic signal $(24.6 \mathrm{~dB})$, represented in dashed line in Fig. 4. It means that the amplifier indeed works in a linear regime for the smallest input energies.

Numerical simulations have been performed to confirm the experimental results. For this purpose, the GNLSE was numerically integrated by using the split step method with experimental parameters taken into account. Higher-order nonlinear effects such as self-steepening have been neglected due to the relatively long pulse duration and the dispersion orders have been limited to the third one. The CFBG was modelled as a purely dispersive element with a dispersion of $350 \mathrm{ps} / \mathrm{nm}$. Note that all nonlinear effects that could occur inside the CFBG have also been neglected due to the extremely low peak power of the pulses. At the output of this dispersive element, we obtained stretched pulses of 193 ps duration at FWHM, in good agreement with analytical relations ${ }^{16}$ and the measured pulse-width after the first pass through the CFBG. These chirped pulses are introduced in the FOPA (modelled through the GNLSE) and the output pulse energies are computed. A very good agreement between numerical simulations (solid line) and experiments (circles) is achieved. The saturation of the amplifier is not surprising since the highest input peak power is about $7 \mathrm{~mW}$ (assuming $1.46 \mathrm{pJ}$ Gaussian pulses of 193 ps duration at FWHM). If a CW signal of this power was introduced in the amplifier, it would also experience a saturated gain of $15 \mathrm{~dB}$, very close to the $12 \mathrm{~dB}$ measured for pulsed signals. This confirms that the lowering of the gain is indeed due to the saturation of the FOPA. 


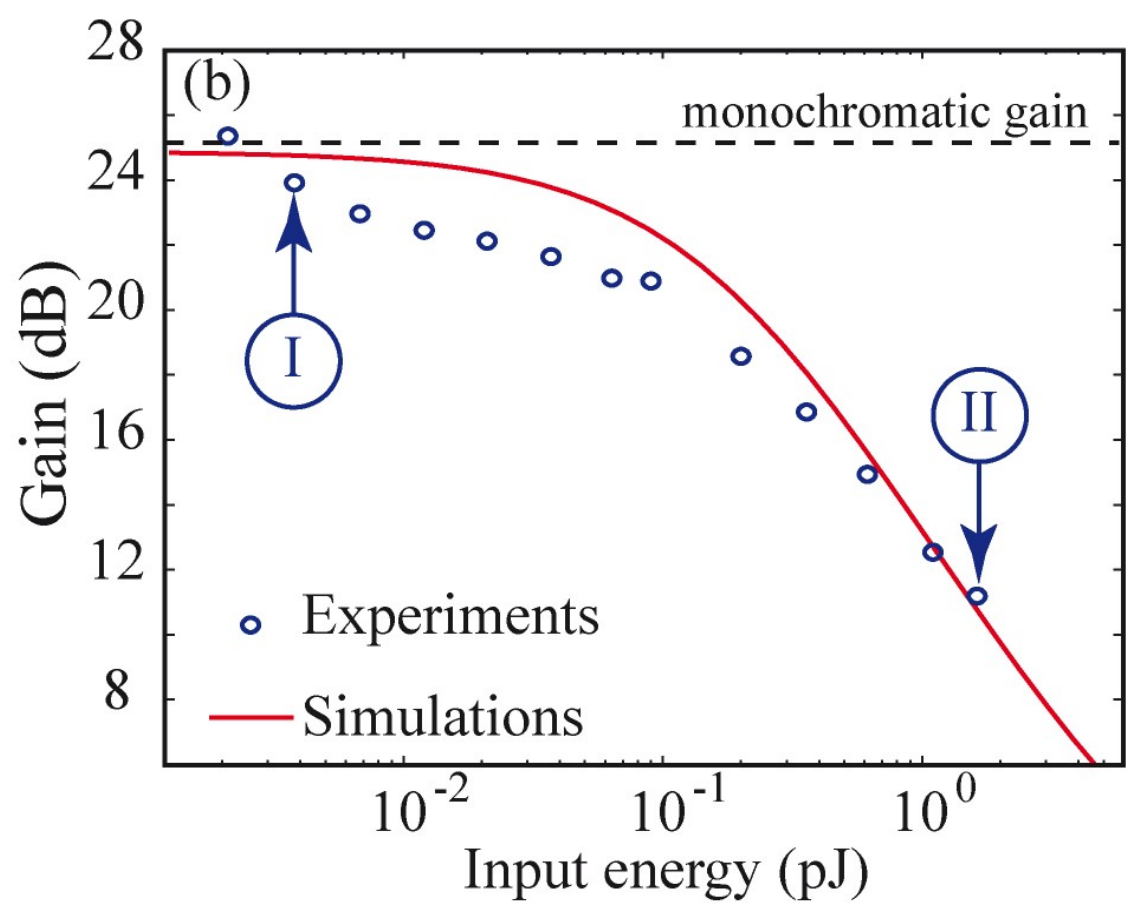

Fig. 4. FOPCPA gain versus signal pulse energy launched into the HNLF for a pump power of $27.6 \mathrm{dBm}$. Cases I and II correspond respectively to the linear and saturated regimes studied in Fig. 5.

In practice, the results presented in Fig. 4 can be further optimized by modifying the FOPA characteristics. To reach this goal, one can play on the stretching ratio. To illustrate this impact, Fig. 5 depicts the numerically computed gain evolutions for 3 different stretching ratios. Keeping the same amplifier, one can see that, with a stretching ratio equal to 600 (obtained by a well designed CFBG), more than $70 \mathrm{~W}$ can be obtained at the FOPA output.

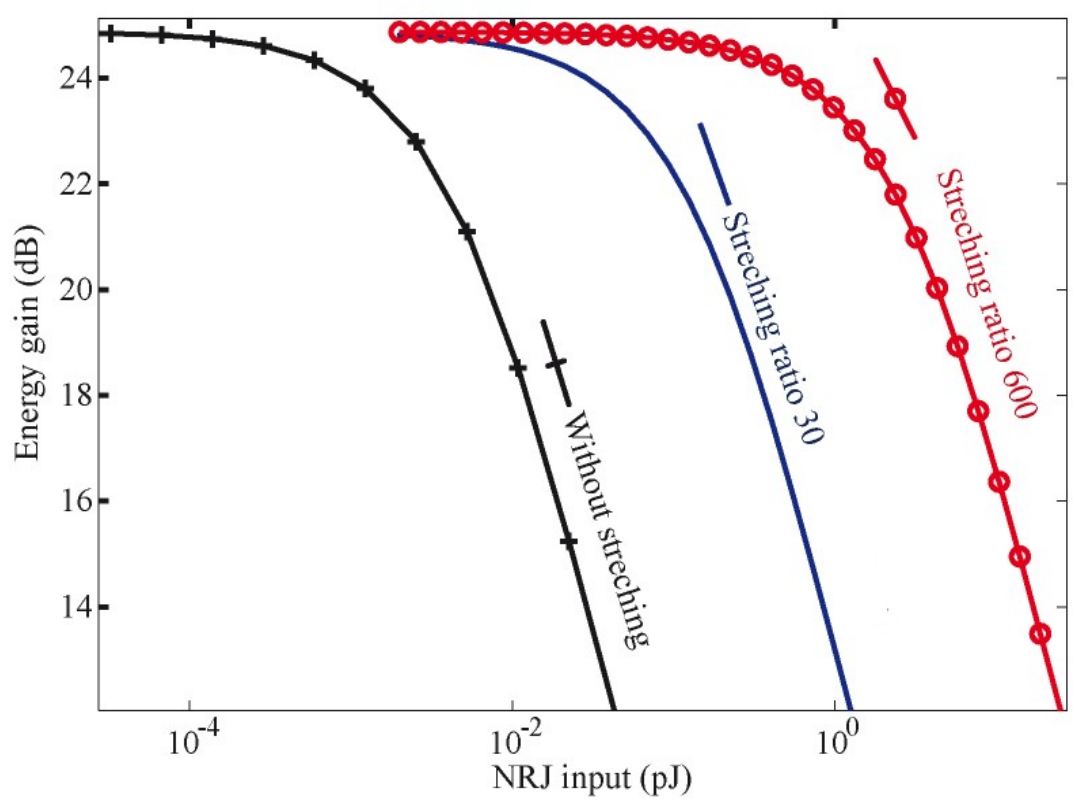

Fig. 5. Numerically computed evolution of the gain versus input energy for 6.4 ps pulses stretched at different ratios. 
A clear spectral signature of the amplifier saturation is shown in Fig. 6, where output spectra corresponding to linear and saturated regimes are represented [cases I and II, respectively, in Fig. 4, for respective input energies of $18.9 \mathrm{fJ}$ and 1.46 $\mathrm{pJ}$ ]. In the linear regime, only one idler wave is generated and the output spectral shape of the signal is quasi-identical to the input one [represented in solid line in Fig. 4]. In the saturated regime, harmonics of the signal and the idler waves are generated in this four-wave mixing process. This multiple-wave interaction associated with the saturation of the amplifier lead to a broadening and a distortion of the output pulses spectrum, as can be seen from Fig. 6. The spectral width is increased up to $1.3 \mathrm{~nm}$, i.e. more than 2 times the initial width $(0.55 \mathrm{~nm})$. From these measurements, one sees that the output signal pulses keep their Gaussian temporal shape in the linear regime, while they are strongly distorted in the saturated one.

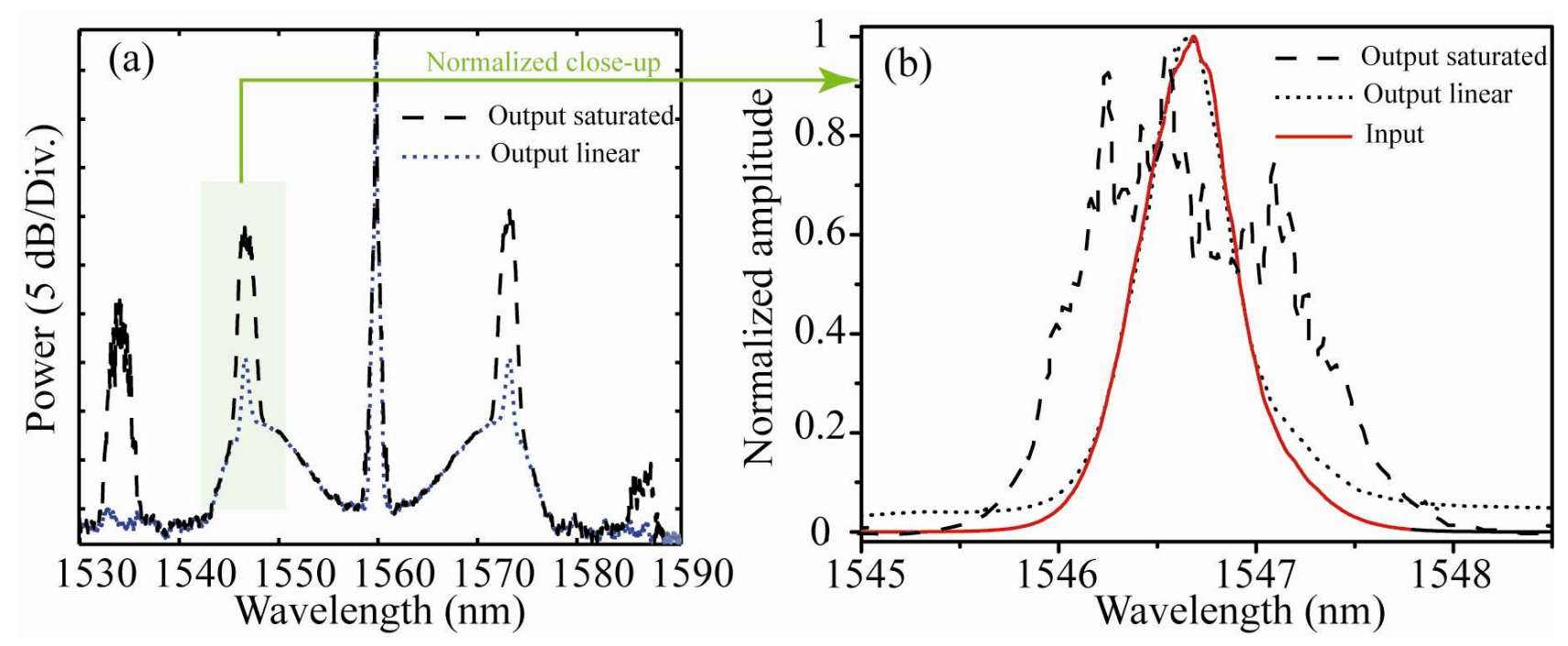

Fig. 6. Optical spectra in the linear [dotted line, corresponding to case I in Fig. 4], and saturated [dashed line, corresponding to case II in Fig. 4] regimes. (a) Whole spectrum and (b) normalized close-up on the signal pulses, plotted in linear scale. The input signal spectrum has been superimposed in solid line in the (b) picture.

Second-order autocorrelation traces of input and output signals after compression for small-signal and deep saturation regimes are represented in Fig. 7. In the small signal regime, input and output pulses profiles are similar, demonstrating that no additional spectral phase is generated during the amplification process. Assuming a Gaussian shape, we deduced from these traces that the signal pulses duration is 6.4 ps at FWHM and that the time-bandwidth product at FWHM is $\Delta v . \Delta t \approx 0.43$, i.e. the pulses are nearly Fourier transform-limited. In saturated amplification, due to the increased spectral width, a pulse shortening is observed. We found a pulse duration of $4.3 \mathrm{ps}$ at FWHM in this case, by assuming a Gaussian shape. Due to the strong distortions of the output signal spectrum we cannot accurately estimate the timebandwidth product, but we expect that the saturation of the amplifier combined with the beating with other waves should lead to additional spectral phase.

\section{CONCLUSIONS}

In this work, we have reported the first experimental demonstration of fiber-based optical parametric chirped pulse amplification. Picosecond Fourier-transform pulses at $1550 \mathrm{~nm}$ were successfully amplified by $24.6 \mathrm{~dB}$ without any spectral or temporal distortions. These performances were reached with a single linearly chirped fiber Bragg grating used for both the stretching and compression operations. The experimental results were confirmed by numerical simulations obtained with the nonlinear Schrödinger equation.

By using photonic crystal fibers one could move the working wavelength around $1 \mu \mathrm{m}$ [13], obtain a broader spectral bandwidth thanks to the high nonlinearity of these fibers, and then scale this technique to the femtosecond regime. 


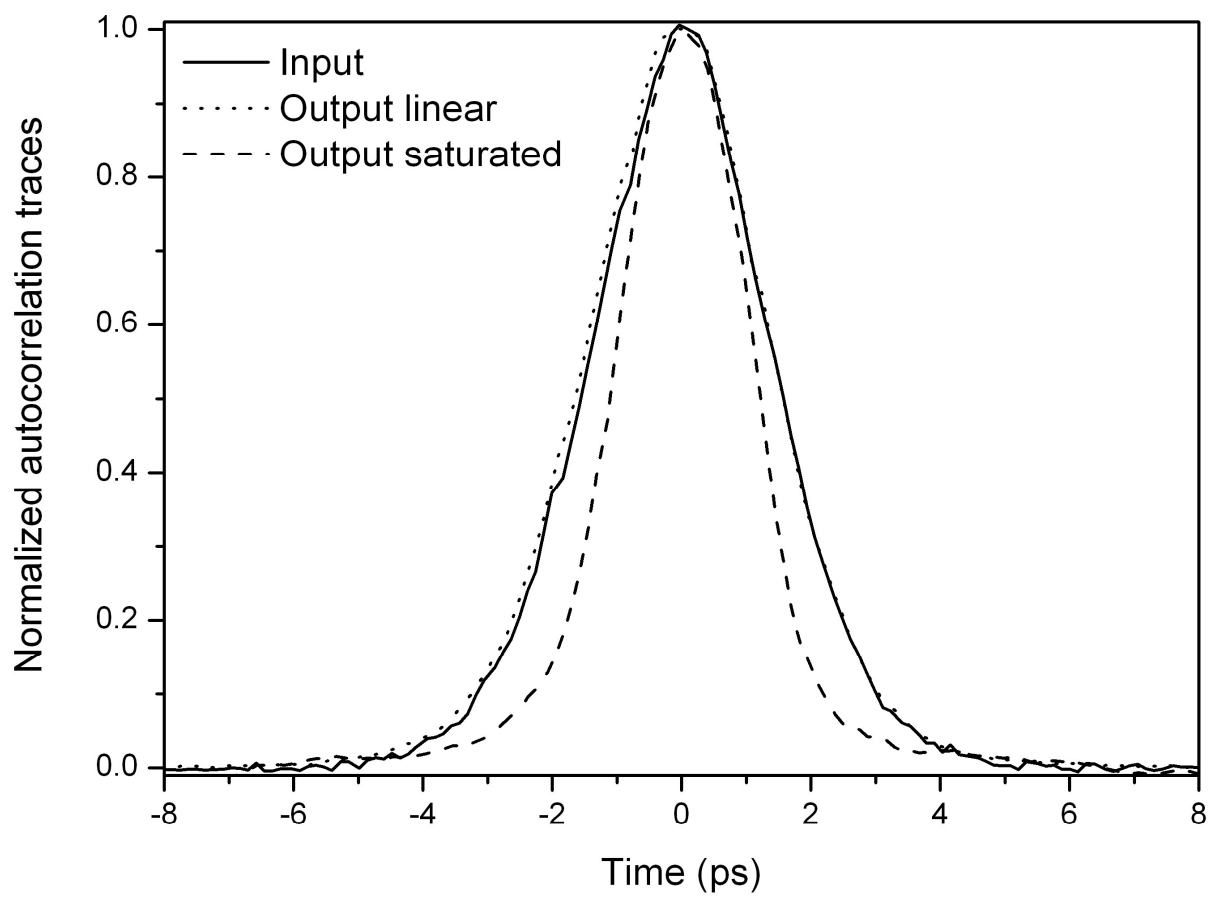

Fig. 7. Measured autocorrelation traces of the input signal (solid line), the amplified signal with 18.9 fJ input energy [dotted line, case I in Fig. 4] and the amplified signal with 1.46 pJ input energy [dashed line, case II in Fig. 4].

\section{ACKNOWLEDGMENT}

Christophe Caucheteur is supported by the FRS - FNRS (Fonds de la Recherche Scientifique - Fonds National de la Recherche Scientifique). C. Caucheteur is supported by the F.R.S.-FNRS. The authors acknowledge the BelSPo IAP 6/10 photonics@be. This work is partially being performed under the auspices of the Conseil Régional du Nord-Pas-deCalais, Conseil Régional d'Aquitaine, French Ministry of Research and European Union, and with the technical supports of the Institut Lasers et Plasmas. We acknowledge Sumitomo for kindly providing the HNLF.

\section{REFERENCES}

1. M. Xu, J. Archambault, J. Reekie, J. Dakin, "Discrimination between strain and temperature using dual-wavelength fibre Bragg grating sensors," Electron. Lett. 30, pp 1085-1087, 1994.

2. D. Strickland, G. Mourou, "Compression of amplified chirped optical pulses," Opt. Commun. 56, pp 219-221, 1985.

3. A. Dubietis, G. Jonusauskas, A. Piskarskas, "Powerful femtosecond pulse generation by chirped and stretched pulse parametric amplification in BBO crystal," Opt. Commun. 88, pp 437-440, 1992.

4. I. N. Ross, P. Matousek, M. Towrie, A. J. Langley, J. L. Collier, "The prospects for ultrashort pulse duration and ultrahigh intensity using optical parametric chirped pulse amplifiers," Opt. Commun. 144, pp 125-133, 1997.

5. A. Dubietis, R. Butkus, A.P. Piskarskas; "Trends in Chirped Pulse Optical Parametric Amplification," IEEE J. Sel. Top. Quantum Electron. 12, pp 163-172, 2006.

6. F. Tavella, A. Marcinkevicious, F. Krausz, "90mJ parametric chirped pulse amplification of 10fs pulses," Opt. Exp. 14, pp 12822-12827, 2006.

7. O.V. Chekhlov, J.L. Collier, I.N. Ross, P.K. Bates, M. Notley, C. Hernandez-gomez, W. Shaikh, C.N. Danson, D. Neely, P. Matousek, S. Hancock, "35 J broadband femtosecond optical parametric chirped pulse amplification system,” Opt. Lett. 31, pp 3665-3667, 2006. 
8. V. Bagnoud, I.A. Begishev, M.J. Guardalben, J. Puth, J. Zuegel "5 Hz, >250 mJ optical parametric chirped-pulse amplifier at $1053 \mathrm{~nm}$," Opt. Lett. 30, pp 1843-1845, 2005.

9. E. Hugonnot, G. Deschaseaux, O. Hartmann, H. Coïc, "Design of PETAL multipetawatt high-energy laser front-end based on optical parametric chirped pulse amplification," Appl. Opt. 46, pp 8181-8187, 2007.

10. J. D. Zuegel, S. Borneis, C. Barty, B. Le Garrec, C. Danson, N. Miyagana, P.K. Rambo, C. Le Blanc, T.J. Kessler, A.W. Schmid, L.J. Waxer, J.H. Kelly, B. Kruschwitz, R. Jungquist, E. Moses, J. Britten, I. Jovanovic, J. Dawson, N. Blanchot, "Laser challenges for fast ignition," in special issue on fast ignition, Fus. Sci. Technol. 49, pp 453-482, 2006.

11. M. Hanna, F. Druon, P. Georges, "Fiber optical parametric chirped-pulse amplification in the femtosecond regime," Opt. Exp. 14, pp 2783-2790, 2006.

12. J. Hansryd, P. Andrekson, "Fiber-Based Optical Parametric Amplifiers and Their Applications," IEEE J. Sel. Top. Quantum Electron. 8, pp 506-520, 2002.

13. M. E. Marhic, Fiber optical parametric amplifiers, oscillators and related devices (Cambridge university press, 2008).

14. T. Sylvestre, A. Kudlinski, A. Mussot, J.F. Gleyze, A. Jolly, H. Maillotte, "Parametric amplification and wavelength conversion in the 1040-1090nm band by use of a photonic crystal fiber," Appl. Phys. Lett. 94, 111104, 2009.

15. A. Mussot, M. Le Parquier, P. Szriftgiser, "Thermal noise for SBS suppression in fiber optical parametric amplifiers," Opt. Commun., doi:10.1016/j.optcom.2010.02.007, 2010.

16. C. Caucheteur, A. Mussot, S. Bette, A. Kudlinski, M. Douay, E. Louvergneaux, P. Mégret, M. Taki, M. GonzalezHerraez, "All-fiber tunable optical delay line,” Opt. Expr. 18, pp 3093-3100, 2010.

17. G. P. Agrawal, Nonlinear Fiber Optics, $4^{\text {th }}$ ed. (Academic Press, 2007). 